\title{
Motion and Force Control of Robot Manipulators
}

\author{
Oussama Khatib and Joel Burdick \\ Artificial Intelligence Laboratory \\ Stanford University Computer Science Department
}

\begin{abstract}
In this paper we present a unified approach for the control of manipulator motions and active forces based on the operational space formulation. The end-effector dynamic model is used in the development of a control system in which the generalized operational space end-effector forces are selected as the command vector. This formulation provides a framework for natural and efficient integration of both end-effector force and motion contral. A "generalized position and force specification matrix" is used for the specification of tasks that involve simultaneous motion and force operations. Flexibility in the force sensor, end-effector, and environment, and problems related to impact are discussed. The real-time operational space control system, COSMOS, has been recently implemented in the NYMPH niultiprocessor system. Results of experiments involving contact and force step input response are presented.
\end{abstract}

\section{Introduction}

The capability of robot systems to perform advanced assembly tasks in unstructured and imprecise environments is strongly dependent on their ability to simultaneously control end-effector motions and active forces. A significant amount of work has been devoted to force control [Whitney 1985]. Accommodation [Whitney 1977], joint compliance [Paul and Shimano 1976], active compliance [Salisbury 1980], and hybrid position/force control [Craig and Raibert 1979] are among the various methods that have been proposed.

These methods have been generally based on kinematic considerations, and were developed within the framework of joint space control systems. Tasks are generally specified in terms of the motions and contact forces of the end-effector. The operational space formulation, which provides an effective means to describe the dynamic behavior of the end-effector, is an efficient and natural framework for the integration of motion and force control.

In this paper we will review the fundamentals of the operational space formulation, and present the means by which motion and force control can be integrated within this framework. The effect of sensor flexibility is analyzed through the use of simple mass/spring models. The behavior of the end-effector during the transition from unconstrained to constrained motion is discussed, and a simple strategy to smoothly control this transition is proposed. A recent multiprocessor implementation of the operational space control system, COSMOS, is described, and experimental results showing the performance for contact and sten response are included.

\section{Operational Space Formulation}

An operational coordinate system is a set $\mathbf{x}$ of $m$ independent end-effector configuration parameters describing its position and orientation in a frame of reference $R_{0}$. For a non-redundant manipulator, the independent parameters $x_{1}, x_{2}, \ldots, x_{m}$ form a complete set of configuration parameters in a domain of the operational space [Khatib 1980] and thus constitute a system of generalized coordinates. The end-effector equations of motion in operational space can be written as [Khatib 1980, Khatib 1983]

$$
\Lambda(\mathbf{x}) \ddot{\mathbf{x}}+\mu(\mathbf{x}, \dot{\mathbf{x}})+\mathbf{p}(\mathbf{x})=\mathbf{F}
$$

where $\Lambda(x)$ designates the kinetic energy matrix, and $\mu(x, \dot{x})$ represents the vector of end-effector centrifugal and Coriolis forces. $\mathbf{p}(\mathbf{x})$ and $\mathbf{F}$ are respectively the gravity and the generalized operational force vectors. With respect to a system of $n$ joint coordinates $\mathbf{q}$, the manipulator equations of motion in joint space can be written in the form

$$
A(\mathbf{q}) \ddot{\mathbf{q}}+\mathbf{b}(\mathbf{q}, \dot{\mathbf{q}})+\mathbf{g}(\mathbf{q})=\mathbf{r}
$$

where $\mathbf{b}(\mathbf{q}, \dot{\mathbf{q}}), \mathbf{g}(\mathbf{q})$, and $\boldsymbol{\Gamma}$, represent the Coriolis and centrifugal, gravity, and generalized forces in joint space; and $\boldsymbol{A ( q )}$ is the $n \times n$ joint space kinetic energy matrix, which is related to $\Lambda(\mathbf{x})$ by

$$
A(\mathbf{q})=J^{T}(\mathbf{q}) \Lambda(\mathbf{x}) J(\mathbf{q})
$$

The extension of the operational space approach to redundant manipulator systems is presented in [Khatib 1980; Khatib 1985].

\section{End-Effector Motion Control}

The control of manipulators in operational space is based on the selection of $\mathbf{F}$ as a command vector. In order to produce this command, specific forces $\boldsymbol{\Gamma}$ must be applied with joint-based actuators. With $q$ representing the vector of $n$ joint coordinates and $J(\mathbf{q})$ the Jacobian matrix, the relationship between $\mathbf{F}$ and the generalized joint forces $\mathbf{F}$ is given by

$$
\Gamma=J^{T}(\mathbf{q}) \mathbf{F}
$$

While in motion, a manipulator end-effector is subject to the inertial coupling, centrifugal, and Coriolis forces. These forces can be compensated for by dynamic decoupling in operational space using the end-effector equations of motion (1). The operational command vector for the end-effector dynamic decoupling and motion control is

$$
\mathbf{F}=\mathbf{F}_{m}+\mathbf{F}_{c c g}
$$


with

$$
\begin{aligned}
\mathbf{F}_{\boldsymbol{m}} & =\Lambda(\mathbf{x}) \mathbf{F}_{m}^{*} \\
\mathbf{F}_{\mathbf{c c g}} & =\mu(\mathbf{x}, \dot{\mathbf{x}})+\mathbf{p}(\mathbf{x}) ;
\end{aligned}
$$

where $\mathbf{F}_{m}^{*}$ is the command vector of the decoupled end-effector. The end-effector becomes equivalent to a single unit mass moving in the $m$-dimensional space. Using equation (4), the joint forces corresponding to the operational command vector $F$ in (5) can be written as

$$
\mathbf{r}=J^{T}(\mathbf{q}) \Lambda(\mathbf{q}) \mathbf{F}_{m}^{*}+\tilde{\mathbf{b}}(\mathbf{q}, \dot{\mathbf{q}})+\mathbf{g}(\mathbf{q})
$$

where $\tilde{\mathbf{b}}(\mathbf{q}, \dot{\mathbf{q}})$ is the vector of joint forces under the mapping into joint space of the end-effector Coriolis and centrifugal force vector $\mu(\mathbf{x}, \dot{\mathbf{x}})$. In order to simplify the notation, the symbol $\Lambda$ has also been used here to designate the kinetic energy matrix when expressed as a function of the joint coordinate vector $\mathbf{q}$. $\widetilde{\mathbf{b}}(\mathbf{q}, \dot{\mathbf{q}})$ is distinct from the vector of centrifugal and Coriolis forces $\mathbf{b}(\mathbf{q}, \dot{\mathbf{q}})$ that arise when viewing the manipulator motion in joint space. These vectors are related by

$$
\tilde{\mathbf{b}}(\mathbf{q}, \dot{\mathbf{q}})=\mathbf{b}(\mathbf{q}, \dot{\mathbf{q}})-J^{T}(\mathbf{q}) \Lambda(\mathbf{q}) \mathbf{h}(\mathbf{q}, \dot{\mathbf{q}}) ;
$$

where

$$
\mathbf{h}(\mathbf{q}, \dot{\mathbf{q}})=\dot{j}(\mathbf{q}) \dot{\mathbf{q}} \text {. }
$$

A useful form of $\widetilde{\mathbf{b}}(\mathbf{q}, \dot{\mathbf{q}})$ for real-time control and dynamic analysis is

$$
\tilde{\mathbf{b}}(\mathbf{q}, \dot{\mathbf{q}})=\tilde{\boldsymbol{B}}(\mathbf{q})[\dot{\mathbf{q}} \dot{\mathbf{q}}]+\tilde{C}(\mathbf{q})\left[\dot{\mathbf{q}}^{2}\right]
$$

where $\tilde{B}(\mathbf{q})$ and $\tilde{C}(\mathbf{q})$ are the $n \times n(n-1) / 2$ and $n \times n$ matrices of the joint forces under the mapping into joint space of the

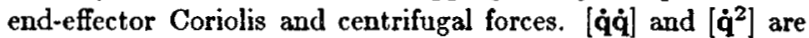
the symbolic notations for the $n(n-1) / 2 \times 1$ and $n \times 1$ column matrices

$$
\begin{aligned}
& {[\dot{q} \dot{q}]=\left[\begin{array}{llll}
\dot{q}_{1} \dot{q}_{2} & \dot{q}_{1} \dot{q}_{3} & \ldots \dot{q}_{n-1} \dot{q}_{n}
\end{array}\right]^{T} ;} \\
& {\left[\dot{\mathbf{q}}^{2}\right]=\left[\begin{array}{lll}
\dot{q}_{1}^{2} & \dot{q}_{2}^{2} & \ldots \dot{q}_{n}^{2}
\end{array}\right]^{T} \text {. }}
\end{aligned}
$$

With the relation (10), the dynamic decoupling of the endeffector can be obtained using the configuration dependent $\mathrm{dy}$ namic coefficients $\Lambda(\mathbf{q}), \widetilde{B}(\mathbf{q}), \widetilde{C}(\mathbf{q})$ and $\mathbf{g}(\mathbf{q})$. By isolating these coefficients, end-effector dynamic decoupling and control can be achieved in a two-level control system architecture. The real-time computation of these coefficients can then be paced by the rate of configuration changes, which is much lower than that of the mechanism dynamics. Furthermore, the rate of computation of the end-effector position can be reduced by integrating an operational position estimator into the control system. Finally, the control system has the following architecture (see Figure 1):

- A low rate parameter evaluation level: updating the endeffector dynamic coefficients, the Jacobian matrix, and the geometric model.

- A high rate servo control level: computing the command vector using the estimator and the updated dynamic coefficients.

\section{Active Force Control}

Tasks are generally described in terms of end-effector motion and applied forces and torques. Let $\boldsymbol{f}_{d}$ and $\tau_{d}$ be the vectors, in the frame of reference $R_{0}\left(O, \mathbf{x}_{0}, \mathbf{y}_{0}, \mathbf{z}_{0}\right)$, of forces and torques that are to be applied by the end-effector. The position of the end-effector can be controlled for motions specified in the subspace orthogonal to $f_{d}$. Let $R_{f}\left(0, \mathbf{x}_{f}, \mathbf{y}_{f}, \mathbf{z}_{f}\right)$ be a frame of reference resulting from $R_{0}$ by a rotation transformation $S_{f}$ such that $\mathbf{z}_{f}$ is in alignment with $f_{d}$. In $R_{f}$, the largest subspace of position control is spanned by $\left\{x_{f}, y_{f}\right\}$. With a task specified in terms of end-effector position control in $\left\{\mathbf{x}_{f}, \mathbf{y}_{f}\right\}$ and force control following $z_{f}$, we associate the task specification matrix [Khatib 1985]

$$
\Sigma_{f}=\left(\begin{array}{lll}
1 & 0 & 0 \\
0 & 1 & 0 \\
0 & 0 & 0
\end{array}\right)
$$

If, in addition, a free motion (zero controlled force) in a direction of the subspace orthogonal to $f_{d}$ is specified, the frame of reference $R_{f}$ can be selected in order to align its $\mathbf{y}_{f}$ axis with that direction, and the corresponding diagonal element in $\Sigma_{f}$ will then be zero. For tasks that specify free motions in the plane orthogonal to $f, \Sigma_{f}$ becomes the $3 \times 3$ zero matrix.

Similarly, let $R_{r}\left(O, x_{r}, y_{r}, z_{r}\right)$ be a frame of reference obtained from $R_{0}\left(0, x_{0}, y_{0}, z_{0}\right)$ by a rotation $S_{\tau}$ that brings $z_{\tau}$ into alignment with the task torque vector $\tau_{d}$. In $R_{r}$, the subspace of end-effector rotations is spanned by $\left\{x_{r}, y_{r}\right\}$. The matrix $\Sigma_{r}$ of task specification associated with this task of rotations and applied torques described in $R_{\boldsymbol{r}}$ is similar to $\boldsymbol{\Sigma}_{\boldsymbol{f}}$. Finally, for general tasks of end-effector position (position and orientation) and applied forces (forces and torques) described in the frame of reference $R_{0}$ we define the generalized position and force specification matrix

$$
\Omega=\left(\begin{array}{cc}
S_{f}^{T} \Sigma_{f} S_{f} & 0 \\
0 & S_{\tau}^{T} \Sigma_{\tau} S_{\tau}
\end{array}\right)
$$

Using $\Omega$, the unified operational command vector for end-effector dynamic decoupling, motion, and active force control can be written as

$$
\mathbf{F}=\mathbf{F}_{\mathbf{m}}+\mathbf{F}_{\mathbf{a}}+\mathbf{F}_{\mathrm{ccg}}
$$

where $\mathbf{F}_{m}, \mathbf{F}_{\mathfrak{a}}$ are the operational command vectors of motion and active force control, given by

$$
\begin{aligned}
\mathbf{F}_{m} & =\Lambda(\mathbf{q}) \Omega \mathbf{F}_{m}^{*} \\
\mathbf{F}_{a} & =\widetilde{\Omega} \mathbf{F}_{a}^{*}
\end{aligned}
$$

where matrix $\widetilde{\Omega}$ is given by

$$
\widetilde{\Omega}=\left(\begin{array}{cc}
S_{f}^{T} \bar{\Sigma}_{f} S_{f} & 0 \\
0 & S_{\tau}^{T} \bar{\Sigma}_{\tau} S_{\tau}
\end{array}\right)
$$

and

$$
\begin{aligned}
& \bar{\Sigma}_{f}=I-\Sigma_{f} ; \\
& \bar{\Sigma}_{r}=I-\Sigma_{r} .
\end{aligned}
$$

The joint force vector corresponding to $F$ in (14), is

$$
\mathbf{\Gamma}=J^{T}(\mathbf{q})\left[\Lambda(\mathbf{q}) \Omega \mathbf{F}_{m}^{*}+\widetilde{\Omega} \mathbf{F}_{a}^{*}\right]+\widetilde{\mathbf{b}}(\mathbf{q}, \dot{\mathbf{q}})+\mathbf{g}(\mathbf{q})
$$

$I$ designates the $3 \times 3$ identity matrix. The control system architecture is shown in Figure 1.

\section{Force Control Compensator}

End-effector forces can be sensed using either wrist force sensors or multi-component finger force sensors. The Stanford force finger sensor is representative of such devices (see Fig- 


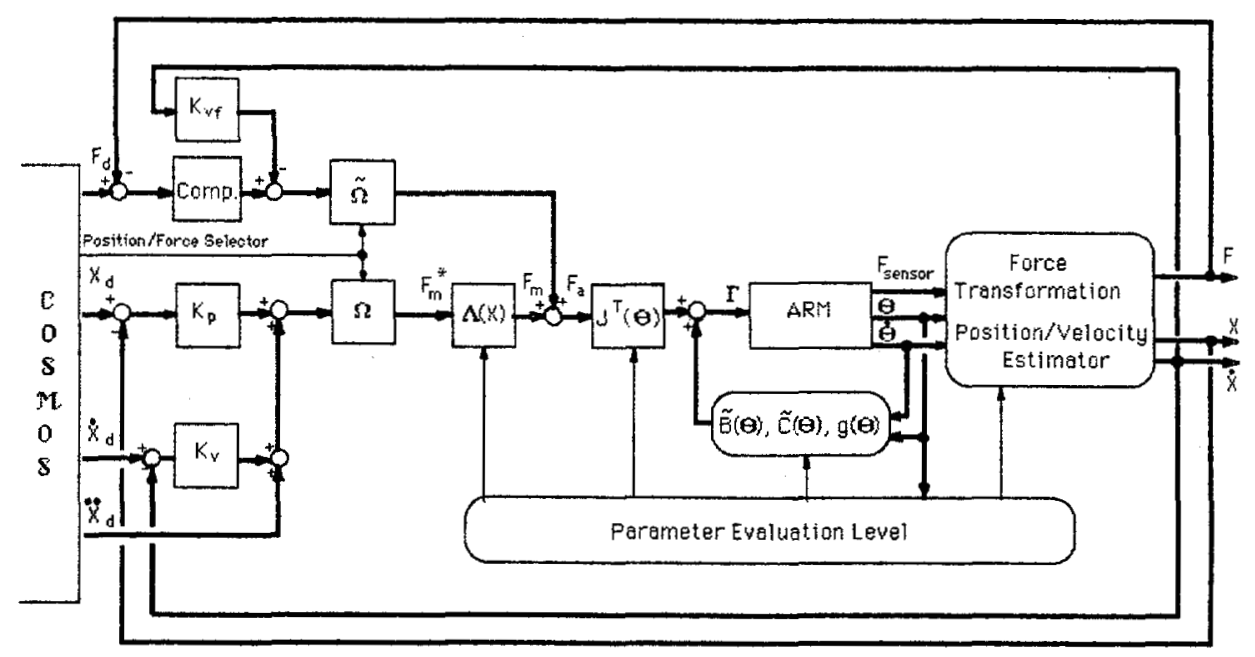

Figure 1. Operational Space Control Architecture

ure 2). This sensor consists of three mechanically decoupled flexible load cells to measure the components of forces applied at the finger tip. Since force sensors are inherently flexible, manipulators can no longer be modeled as rigid bodies when instrumented with these devices.

The dynamic behavior of the end-effector/sensor system is complex, but can be approximately modeled as a mass and spring oscillator in the $m$-dimensional space. At the level of the decoupled end-effector, the dynamic behavior of the end-effector/sensor mechanism during contact with an object, in a given direction $\mathrm{z}_{f}$, can be represented by the mass and spring system shown in Figure 3, where the finger tip mass has been neglected.

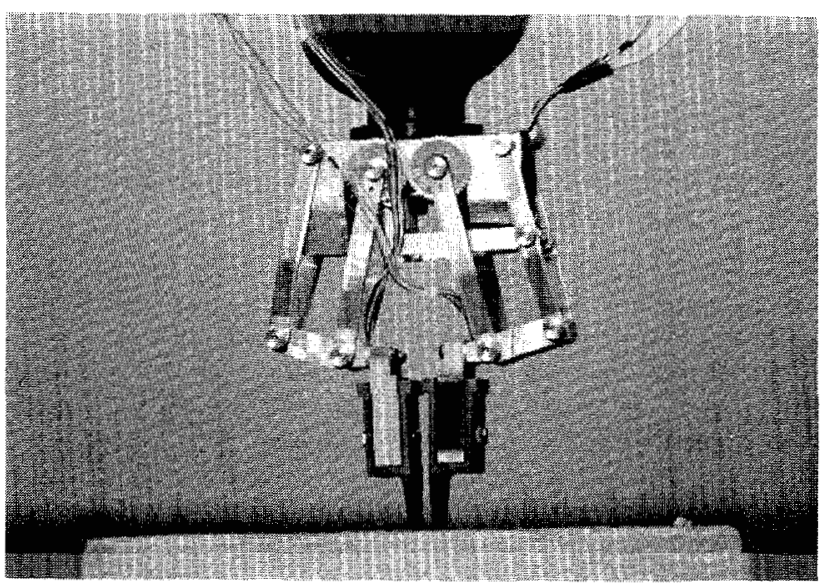

Figure 2. Stanford Force Sensing Fingers

By an appropriate selection of the coordinate origin, the equation of motion along the $z_{f}$ direction is

$$
m_{z_{f}} \ddot{z}_{f}+k_{z_{f}} z_{f}=f_{z_{a}}
$$

where

$$
\begin{aligned}
& m_{x_{y}}=\text { effective mass of the arm at a given configuration } \\
& \quad \text { in the } \mathbf{z}_{\mathrm{f}} \text { direction } \\
& f_{x_{\alpha}}=\text { the operational control force }
\end{aligned}
$$

$$
\begin{aligned}
k_{x_{j}}= & \text { the effective stiffness of the sensor and environment } \\
& \text { in the } \mathbf{z}_{f} \text { direction } \\
& =\left(1 / k_{t}+1 / k_{\varepsilon}\right)^{-1}
\end{aligned}
$$

Where $k_{,}$is the effective stiffnesses of the force sensor and $k_{c}$ is the effective stiffness of the environment in the $z_{f}$ direction. The effective mass of the arm, $m_{z_{f}}$, which varies with the direction and configuration of the manipulator is given by

$$
m_{z_{f}}=\mathbf{u}^{T} \mathbf{\Lambda}(\mathbf{q}) \mathbf{u}
$$

where

$$
\mathbf{u}=\left[\begin{array}{ll}
\mathbf{z}_{f}^{T} & 0
\end{array}\right]^{T}
$$

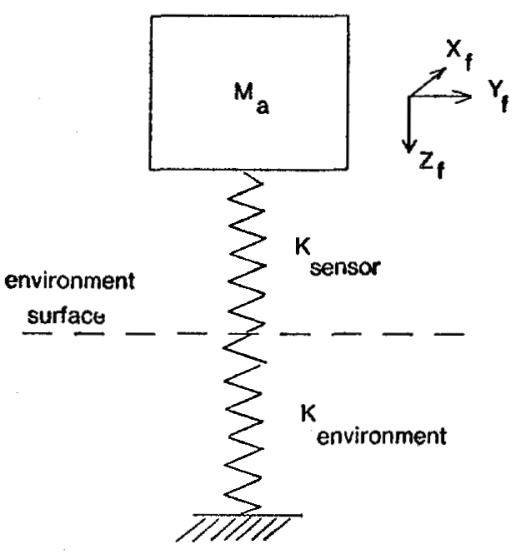

Figure 3. Simplified End-effector/Sensor Model

Based on this simplified model, force control along the $z_{f}$ direction can be achieved with the control structure

$$
f_{z_{a}}=f_{x_{d}}+k_{f}\left(f_{x_{d}}-f_{z}\right)-m_{x_{f}} k_{v_{f}} z_{f} ;
$$

where $f_{z_{d}}$ is the desired applied force, and $k_{f}$ and $k_{v}$ are the force error and velocity damping gains. In this simplified model, velocity damping can also be achieved by force derivative feedback, since velocity is proportional to force derivative $\left(\dot{f_{x}}=k_{x}, \dot{z}_{f}\right)$. 
The corresponding operational space command vector, $\mathbf{F}_{\mathrm{a}}$ in equation (15), for the control of active forces is

$$
\mathbf{F}_{a}=\tilde{\Omega}\left(\mathbf{F}_{d}+K_{f}\left(\mathbf{F}_{d}-\mathbf{F}_{\boldsymbol{b}}\right)\right)-K_{v_{f}} \mathbf{A} \tilde{\mathbf{\Omega}} \dot{\mathbf{x}}
$$

where $K_{f}$ and $K_{v_{f}}$ are diagonal matrices with components $k_{f}$ and $k_{v}$.

\section{End-Effector Behavior During Impact}

The control of a manipulator during transition from free to constrained motion of the end-effector is an important operation in tasks that involve active force control. For robot manipulator systems operating in an unstructured environment with position uncertainties, this transition can be expected to occur at nonzero velocity. A large amount of energy can be involved during impact. The dissipation of that energy is essential in order to achieve stable contact and avoid bounces and vibrations.

The forces that arise during contact are a function of contact geometry, normal velocities, and the stiffnesses of the nating parts. The magnitude of these forces increases with impact velocity. An effective strategy which allows rapid response, while avoiding bounces and minimizing force overshoots, consists of introducing a transitory stage of control during the first instants of impact. The "impact transition" control stage is primarily aimed at fast dissipation of the excessive impact energy. This dissipation can be simply obtained by pure velocity damping

$$
\mathbf{F}_{a}=-K_{v} \Lambda(\mathbf{q}) \tilde{\Omega} \dot{\mathbf{x}} .
$$

The duration of the impact transition control will be a function of the impact velocity and the limitations on damping gains.

\section{COSMOS System}

This approach has been implemented in an experimental manipulator programming and control system, COSMOS. COSMOS has recently been implemented in the NYMPH multiprocessor system [Chen et. al. 1986]. COSMOS had previously been implemented in a parallel processing system that used a PDP 11/45 and a PDP 11/60 minicomputer. This implementation was inadequate for force control research and development due to limitations in memory and real time interprocessor communication.

The NYMPH system consists of multiple National Semiconductor 32016 microprocessors and a SUN Microsystems workstation integrated on an Intel multibus. The 32016 processors perform the real time computations, while the SUN, via the V-kernel operating system, performs user and system interface functions.

As mentioned in section (3), the computation of the operational space control is conveniently decomposed into a "high" and "low" and level, which can be implemented on parallel processors. Because of the decoupled nature of the control, each of these levels can be furthered divided to extract more parallelism. In the current three processor COSMOS implementation the low level servo is divided so that the position and orientation servos are computed on separate processors. The position and orientation kinematics and dynamics, and the run time program decoding functions are implemented in the third processor. With this arrangement, low level servo rates of $200 \mathrm{~Hz}$ and high level dynamics rates of $100 \mathrm{~Hz}$ have been achieved. A five processor COSMOS implementation is currently in progress, and will divide the high level position and orientation dynamic computations and the programming functions into three processors (see Figure 4).

$$
\text { High Level Low Level }
$$

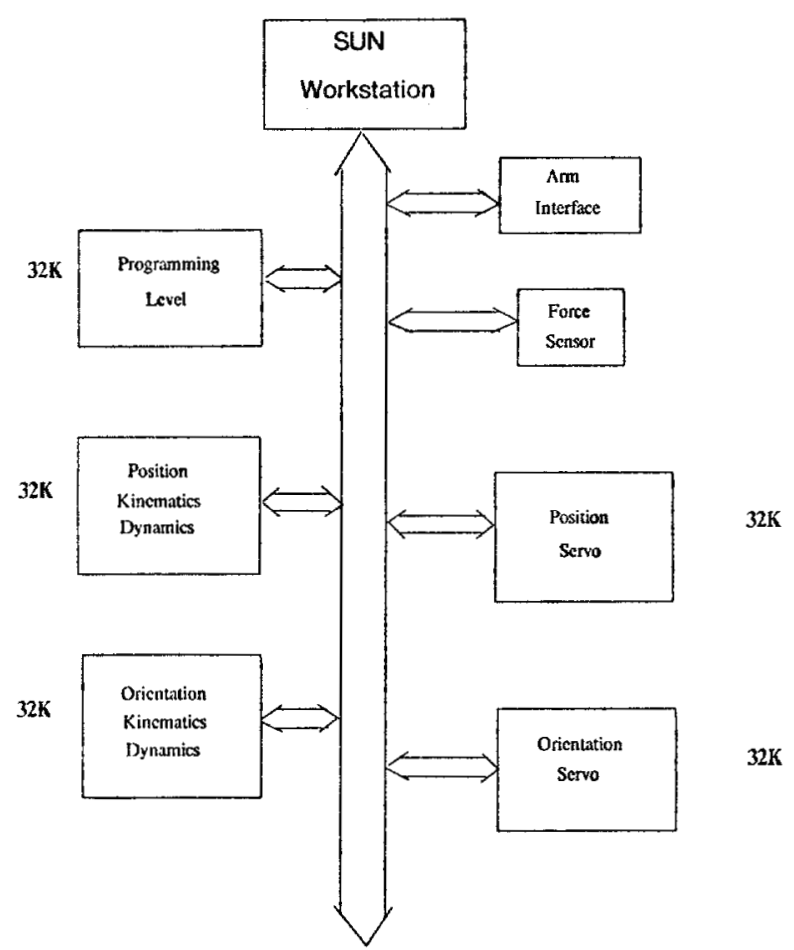

Figure 4. COSMOS Multiprocessor Architecture

\section{Experimental Results}

COSMOS has been used for motion and force control of a PUMA 560 manipulator. Both a force wrist (Stanford/Schieneman design) and the Stanford finger force sensors have been used to provide end-effector force feedback.

With finger force sensing, experiments including impact and step response have been conducted. Figure 5 shows the contact force time response from a typical experiment. In this experiment, the sequence of operations consists of making contact with a rigid surface, followed immediatedly by a square wave force input of $-20.0-40.0$, and -20.0 ounces. The end-effector velocity in the direction normal to the contact surface was 4.0 inches/second at impact.

The velocity at impact has been transferred into a force overshoot. For the first 0.10 seconds after impact the transition control strategy of equation (24) was successfully employed to avoid bounce and oscillations, while thereafter the force control law described in equation (23) was used to servo end-effector forces. The rise time in response to the step inputs is 18 milliseconds, while the steady state force error in all cases is $\mathbf{2 . 4}$ ounces.

Similar experiments have been conducted using wrist force sensing feedback. The response to a square wave input is shown in Figure 6. The steady state force error was an average of 3.2 ounces while the rise time was 20 milliseconds. 


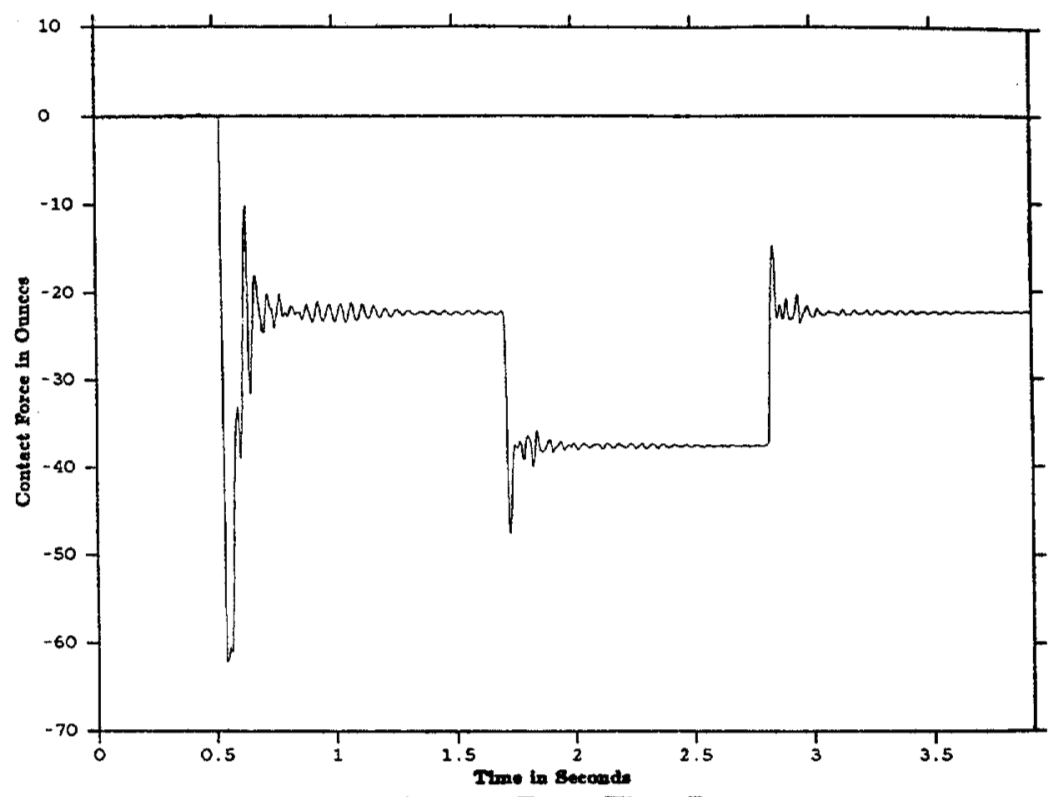

Figure 5. Contact Force Time Response

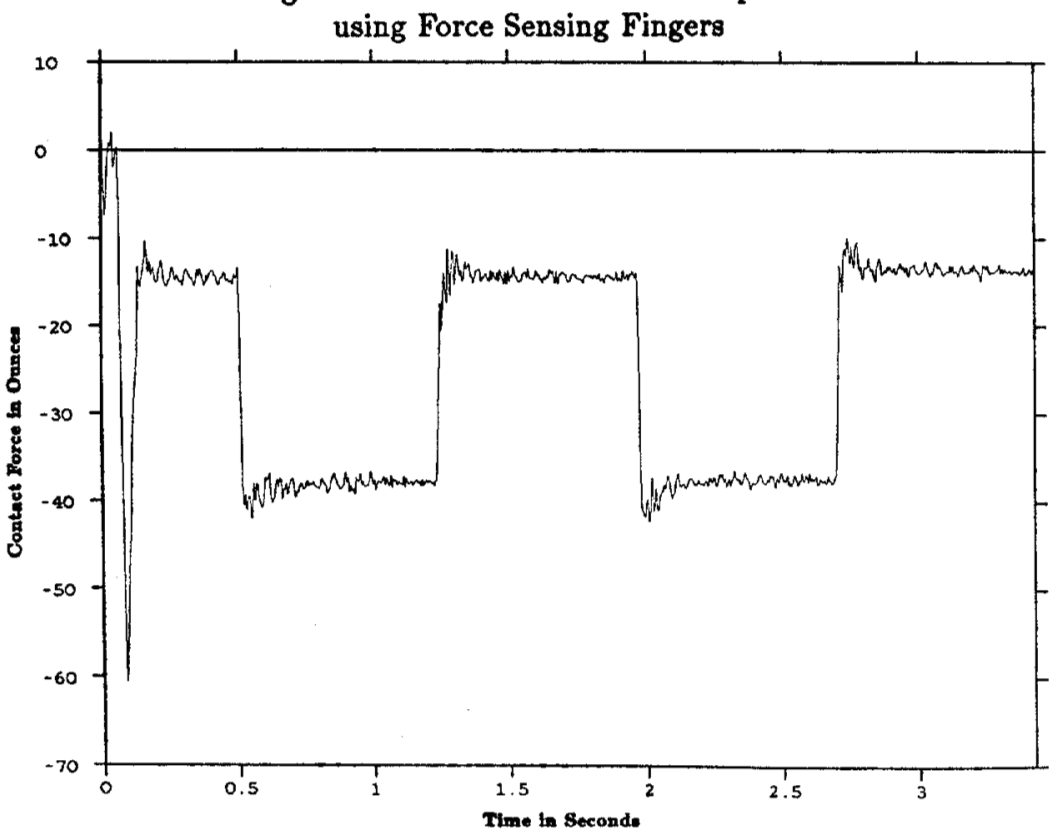

Figure 6. Contact Force Time Response using Force Sensing Wrist

The force sensing fingers used in these experiments have a resolution of 2.0 grams and a maximum force measurement of 5.5 pounds. In contrast, the resolution of the force sensing wrist is 15 grams; and a maximum force of 40.0 pounds can be measured. The tip of the force finger sensor has negligible mass, while the mass of the end-effector supported by the force wrist amounts to nearly 3.0 pounds. In conjunction with the complex flexing structure used in the wrist, the presence of this large mass leads to complex vibrations of significant amplitude that can not be modelled by a simple mass/spring system.

\section{Summary and Discussion}

This approach to motion and force control is based on the precise control of joint torques. However, for most industrial ma- nipulators, such as the PUMA 560, the control of joint torques is difficult to achieve due to gear cogging, friction, stiction, and backlash in the actuator transmission mechanisms. Typically, the actuators of the PUMA used in these experiments exhibit a static dead band that can be as high as $20 \%$ of their maximum torque output. The performance achieved in the experimental results has been obtained despite these limitations.

This level of performance is the result of the nearly perfect dynamic decoupling of the manipulator end-effector motions and forces obtained from the operational space approach. This performance has been further enhanced by the development of an efficient and accurate dynamic model of the PUMA [Burdick 1986], and by the accurate identification of the PUMA dynamic parameters [Armstrong, Khatib, and Burdick 1986]. 
At the level of the decoupled end-effector, a simple mass/spring model has been used in formulating the active force control command vector. However, these simple models are only an approximation to the real behavior of manipulators during force controlled operation. Flexibilities and nonlinearities in the manipulator links, joint actuator systems, end-effector gripping devices, and force sensors will contribute additional flexibility and resonant modes to the system. Some of these higher order unmodelled modes can be observed in the force time response shown in Figure 6. These unmodelled modes limit the level of performance that can be obtained with a control system based on simplified modelling.

Higher force control performance can be achieved by a two level approach. At the level of the end-effector/sensor system, this involves accurate modeling and identification of the endeffector/sensor modes, and the design of a higher order and a more robust force control compensator. Considering the high frequencies involved during force controlled operations, higher servo rates and discrete digital compensator design are also necessary for increased performance.

And, at the level of the articulated mechanisms, the limitations imposed by the flexibilities and nonlinearities in the actuator transmission systems should also be compensated for. These limitations can be minimized by the use of joint torque feedback compensation using joint torque sensing [Luh, Fisher, and Paul 1981], [Pfeffer, Khatib, and Hake 1986].

However, the level performance that can be obtained by retrofitting typical industrial manipulators designed for position control operations will remain limited. A higher level of performance can only be achieved by a new design of mechanisms based on the requirements of manipulator force control. Actuator/transmission systerns [Asada and Youcef-Toumi 1983], end-effector dynamic characteristics, [Khatib and Burdick 1985], redundancy [Hollerbach 1984] and micro-manipulation ability for precise motion and fine force control are among the various issues that should be considered in force controlled manipulator design.

\section{Acknowledgements}

The authors would like to thank Brian Armstrong and Ron Fearing for their invaluable technical assistance. Support for this work has been provided by the National Science Foundation under contract MEA80-19628, by a grant from the Systems Development Foundation, and by DARPA through the Intelligent Task Automation Project, contract F 33615-82-C-5092.

\section{References}

Armstrong, B., Khatib, O., and Burdick, J. 1986 (April). "The Explicit Dynamic Model and Inertial Parameters of the PUMA 560 Arm." To be presented at the 1986 IEEE International Conference on Robotics and Automation, San Fransisco, CA.

Asada, H. and Youcef-Toumi, K. 1983 (June). Analysis and Design of Semi-Direct-Drive Robot Arms. 1983 American Control Conference. pp. 757-764. San Francisco.

Burdick, J. 1986 (April). "An Algorithm for Generation of Efficient Manipulator Dynamic Equations." To be presented at the 1986 IEEE International Conference on Robotics and Automation, San Fransisco, CA.
Chen, B., Fearing, R., Armstrong, B., and Burdick, J. 1986 (April). "Nymph: A Multiprocessor for Manipulation Applications." To be presented at the 1986 IEEE International Conference on Robotics and Automation, San Fransisco, CA.

Craig, J.J. and Raibert, M. 1979. A Systematic Method for Hybrid Position/Force Control of a Manipulator. Proceedings 1979 IEEE Computer Software Applications Conference, Chicago.

Hollerbach, J.M. 1984 (August). Optimum Kinematic Design for a Seven Degree of Freedom Manipulator. Prep. of the 2nd International Symposium of Robotics Research, Kyoto, Japan, pp. 349-356.

Khatib, O. 1980 (December). Commande Dynamique dans l'Espace Opérationnel des Robots Manipulateurs en Présence d'Obstacles. Thése de Docteur-Ingénieur. École Nationale Supérieure de l'Aéronautique et de l'Espace, Toulouse, France.

Khatib, O. 1983 (December 15-20). Dynamic Control of Manipulators in Operational Space. Sixth CISM-IFToMM Congress on Theory of Machines and Mechanisms, pp. 1128-1131, New Delhi, India (Wiley, New Delhi).

Khatib, O. Burdick, J. and Armstrong, B. 1985. Robotics in Three Acts - Part II (Film). Artificial Intelligence Laboratory, Stanford University.

Khatib, O. 1985 (October). The Operational Space Formulation in the Analysis, Design, and Control of Manipulators. The Third International Symposium of Rabotics Research, Paris, France.

Khatib, O. and Burdick, J. 1985 (November). Dynamic Optimization in Manipulator Design: The Operational Space Formulation. The 1985 ASME Winter Annual Meeting, Miami.

Luh, J.Y.S., Fisher, W.D., and Paul, R.P. 1981 (December). Joint Torque Control by a Direct Feedback for Industrial Robot. Proc. 20th IEEE Conference on Decision and Control, San Diego, pp. 265-271.

Paul, R.P. and Shimano, B. 1976 (July). Compliance and Control. Proceedings of the Joint Automatic Control Conierence. pp. 694-699. Purdue University.

Pfeffer, L. Khatib, O. and Hake, J. 1986 (June) Joint Torque Sensory Feedback in the Control of a PUMA Masipulator. ('To be presented at.) The 1986 American Control Conference, Seattle.

Salisbury, J.K. 1980 (December) Active Stiffness Control of a Manipulator in Cartesian Coordinates. 19th IEEE Conference on Decision and Control, Albuquerque, New Mexico.

Whitney, D.E. 1977 (June). Force Feedback Control of Manipulator Fine Motions. ASME, Journal of Dynamir Systems, Measurement, and Control, pp. 91-97.

Whitney, D.E. 1985 (March). Historical Perspective and State of the Art in Robot Force Control. Proceedings of the 1985 IEEE International Conference on Robotics and Automation, pp. 262-268, St. Louis. 